

\title{
Emergence of an enslaved phononic bandgap in a non-equilibrium pseudo-crystal
}

Nicolas Bachelard, Chad Ropp, Marc Dubois, Rongkuo Zhao, Yuan Wang, Xiang Y. Zhang

\section{- To cite this version:}

Nicolas Bachelard, Chad Ropp, Marc Dubois, Rongkuo Zhao, Yuan Wang, et al.. Emergence of an enslaved phononic bandgap in a non-equilibrium pseudo-crystal. Nature Materials, 2017, 16 (8), pp.808-813. 10.1038/NMAT4920 . hal-02145725

\section{HAL Id: hal-02145725 \\ https://hal.science/hal-02145725}

Submitted on 6 Jun 2019

HAL is a multi-disciplinary open access archive for the deposit and dissemination of scientific research documents, whether they are published or not. The documents may come from teaching and research institutions in France or abroad, or from public or private research centers.
L'archive ouverte pluridisciplinaire HAL, est destinée au dépôt et à la diffusion de documents scientifiques de niveau recherche, publiés ou non, émanant des établissements d'enseignement et de recherche français ou étrangers, des laboratoires publics ou privés. 


\section{Emergence of an enslaved phononic bandgap in a non- equilibrium pseudo-crystal}

Nicolas Bachelard ${ }^{1+}$, Chad Ropp $^{1+}$, Marc Dubois ${ }^{1}$, Rongkuo Zhao ${ }^{1}$, Yuan Wang ${ }^{1,2}$, Xiang Zhang $^{1,2,3 *}$

${ }^{1}$ NSF Nanoscale Science and Engineering Center, 3112 Etcheverry Hall, University of California, Berkeley, California 94720, USA. ${ }^{2}$ Materials Sciences Division, Lawrence Berkeley National Laboratory, 1 Cyclotron Road, Berkeley, California 94720, USA. ${ }^{3}$ Department of Physics, King Abdulaziz University, Jeddah 21589, Saudi Arabia.

${ }^{\dagger}$ These authors contributed equally to this work.

*email: xiang@berkeley.edu

Material systems that reside far from thermodynamic equilibrium have the potential to exhibit dynamic properties and behaviours resembling those of living organisms. Here we realize a non-equilibrium material characterized by a bandgap whose edge is enslaved to the wavelength of an external coherent drive. The structure dynamically self-assembles into an unconventional pseudo-crystal geometry that equally distributes momentum across elements. The emergent bandgap is bestowed with lifelike properties, such as the ability to self-heal to perturbations and adapt to sudden changes in the drive. We derive an exact analytical solution for both the spatial organization and the bandgap features, revealing the mechanism for enslavement. This work presents a framework for conceiving lifelike nonequilibrium materials and emphasizes the potential for the dynamic imprinting of material properties through external degrees of freedom. 
Materials in nature spontaneously form through microscopic and collective interactions. This self-organization process is often a source of wonder and a catalyst for scientific inspiration. By understanding crystal growth, people have been able to artificially synthesize materials with high purity ${ }^{1}$ and subsequently invent new composite ${ }^{2,3}$ and crystalline forms ${ }^{4-6}$. Crystals are examples of the rigid structures that form by static self-assembly, the process through which order arises in an equilibrium state ${ }^{7}$. Still, nature shows us that order can arise far from thermodynamic equilibrium as seen in living cells ${ }^{8}$, the swarming and flocking of animals ${ }^{9-12}$, and even weather patterns ${ }^{13}$. These systems arise and are sustained outside of thermodynamic equilibrium through the collective dissipation of energy (i.e. irreversible loss) in a process known as dynamic self-assembly ${ }^{14}$. Remarkably, this self-sustaining process produces structures exhibiting lifelike behaviours ${ }^{15}$, such as the ability to self-heal ${ }^{15}$ [remove and cite osterman], self-adapt to changes in the drive ${ }^{16}$ [also cite solis, Snezhko 2006, Snezhko 2011], and even selfreplicate $^{17}$. Non-equilibrium systems provide a foundation for creating artificial structures that are inspired by nature and mimic living organisms ${ }^{20-24}$ [also cite solis, Snezhko 2009].

Materials research is ultimately devoted to the study of physical properties. For crystalline materials, which reside in thermodynamic equilibrium, macroscopic properties often result from the microscopic order-for example photonic bandgaps resulting from the periodicity of opals $^{25,26}$ or phononic bandgaps resulting from mode hybridization. The question arises whether these properties can be imprinted directly into a material without requiring the systematic engineering of the material's microscopic structure. Towards this end, nonequilibrium materials can provide a solution. Non-equilibrium systems are inherently open to their environment and the necessary input of energy offers a route for the external modification of material responses. This open channel has been utilized to ... and been utilized to tune the geometry of non-equilibrium structures, for example by changing the flow rate ${ }^{18}$ or frequency $^{16,19}$ of the drive. However, beyond the tuning of geometry, exploiting this channel to dynamically imprint physical properties into non-equilibrium materials has never been investigated. Such ability could enable external degrees of freedom to direct self-organization and simultaneously encode macroscopic properties. 
In this article, we report the first demonstration of a non-equilibrium material with emergent bandgap properties that are enslaved to the wavelength of an external coherent source. The system consists of an array of scattering particles sitting in a viscous liquid and confined within a one-dimensional single-mode waveguide. These particles dynamically self-assemble towards a configuration that equally distributes momentum along the structure. The resulting order corresponds to an unconventional pseudo-crystal exhibiting a phononic bandgap, whose band edge is enslaved to the wavelength of an acoustic source. The structure resides far from equilibrium, reaching a steady state that moves forward in time and constantly dissipates energy. Remarkably, the lifelike behaviours often observed in non-equilibrium structures are here bestowed to the emergent bandgap itself. This is manifested in its ability to self-heal to mechanical perturbations and self-adapt to changes in the drive wavelength. Seldom achieved for non-equilibrium systems ${ }^{27}$, we are able to derive an exact analytical solution for the nonequilibrium steady state. This solution is confirmed experimentally and emphasizes the central role of wave coherence in bandgap enslavement. In addition, our design offers the ability to investigate the transient process of dynamic self-assembly, from which we experimentally confirm dynamic attraction and subsequent phase-space collapse. Finally, we stress that our analytic solution is completely general and applicable to any classical wave system.

\section{Emergence of an enslaved bandgap.}

Our experiment consists of a single-mode acoustic waveguide filled with 12 mobile particles that sit in the meniscus of a viscous liquid (see Methods). These particles are put in motion by the continuous acoustic pressure exerted by a coherent and unidirectional drive field (Fig. 1a). The system dynamically self-assembles into a non-equilibrium structure, which coincides with the emergence of a phononic bandgap with its higher-wavelength band edge enslaved to $\lambda_{0}$, the wavelength of the source (Fig. $1 \mathrm{~b}$ and Supplementary Video 1 ). At $t=0$ the source is turned on and the particles begin to move in various directions. Eventually, they collectively organize into a structure that moves forward in time and continuously dissipates kinetic energy through viscous friction. This dissipation not only ensures far from thermodynamic equilibrium operation, but also the stability of any accessible steady state by enforcing overdamped particle 
motion (see Methods). Remarkably, the enslaved bandgap emerges regardless of the initial random placement of particles, even when the steady-state organizations are different (Supplementary Fig. 1).

The emerging bandgap exhibits several lifelike behaviours-it self-heals to mechanical perturbations (Fig. 1c and Supplementary Video 2) and re-emerges at different wavelengths in response to sudden changes in the drive (Fig. 1d and Supplementary Video 3). Although geometric structures arising through dynamic self-assembly often exhibit self-healing and selfadaptive characteristics ${ }^{15}$, here these lifelike properties are readily bestowed to the bandgap feature itself.

\section{Pseudo-crystal formation.}

The mechanisms that govern the dynamic self-assembly in our system are revealed by monitoring the trajectories of individual particles (see Methods). In contrast to conventional bandgap materials emerging from crystalline order ${ }^{28}$, our structure appears pseudo-crystalline as a consequence of the coherent and equal distribution of momentum amongst the particles (Fig. 2a). To better visualize this pseudo-crystalline order, we track the particles' positions during the self-assembly process (see Methods) and decompose their centre-to-centre distance as $x_{n}-x_{n-1}=D_{S}+p_{n} \lambda_{0} / 2+d_{n}$, where $D_{S}$ is the width of each particle, $p_{n}$ is a distribution of positive integers, and $d_{n}$ is the residual separation between particles, modulo $\lambda_{0} / 2$. In this definition, $n \in[1, N]$ for $x_{n}$, while $n \in[2, N]$ for $p_{n}$ and $d_{n}$, where $N$ is the number of particles.

The system evolves towards a non-equilibrium steady state with a non-uniform $p_{n}$ distribution, which describes the pseudo-crystal geometry (Fig. 2a). This geometry fulfils the phase requirement imposed by the coherent field, producing the bandgap response. Self-organization is mediated by the feedback between particle motion and wave scattering. The coherent field exerts acoustic pressure on the particles, whose motion in turn modifies the scattered field. Particle interactions are thus mediated by the field, whose intensity interferences are unchanged by additional $p_{n} \lambda_{0} / 2$ separations. The preservation of $\lambda_{0}$-coherence is confirmed 
experimentally by the $\pi$-phase degeneracy observed in our structure. Depending on the initial configuration, any distribution of $p_{n}$ can be reached (Supplementary Fig. 1), indicating that our system can self-assemble into many equivalent pseudo-crystalline geometries with similar bandgap responses. While dynamic self-assembly commonly arises through hydrodynamic interactions $^{16,18,21,23}$, here the assembly is entirely mediated by the coherent field, which produces multiple interaction length scales depending on $p_{n}$. Since particles are only weakly absorbing (see Methods) and our system is confined into a single-mode waveguide, the scattering into higher-order modes is precluded and the coherence is preserved along the structure. By preserving mode coherence, our system is in contrast to other wave-based approaches for self-assembly, such as optical binding ${ }^{29}$. These approaches utilize scattering into multiple spatial modes in order to produce long distance coupling between particles ${ }^{30,31}$, but as a result lose the spatial coherence required for bandgap emergence.

The time evolution of our system is characterized by the attraction towards a configuration with a nearly constant, but monotonically decreasing residual spacing, $d_{n}$ (Fig. $2 \mathrm{~b}$ and inset). This organization is in further contrast to conventional bandgap crystals that, being periodic, would have a constant $d_{n}$. The asymmetry observed in $d_{n}$ originates from the open boundary conditions (see Methods) and the unidirectional transfer of momentum from the source to the ensemble, which produces the overall one-way motion. This asymmetry is also observed in the evolution of $d_{n}$, as displayed by the slower temporal response and pronounced oscillations for larger $n$ (Supplementary Fig. 2). Snapshots of $d_{n}$ at different times also reveal the connection between particle order and bandgap self-healing and self-adaptability. Self-healing of the bandgap coincides with self-healing of $d_{n}$ (Fig. 2c), while bandgap emergence at different wavelengths is reflected by an overall shift in $d_{n}$ (Fig. $2 \mathrm{~d}$ ).

\section{Theoretical description of the emergent bandgap.}

At steady state the system self-organizes into a pseudo-crystalline geometry that equally distributes momentum across all particles. Due to system confinement to a single-mode waveguide, the spatiotemporal coherence of the drive field is maintained throughout multiple scattering events. The coherence enforces a steady-state condition in which each particle 
experiences the same pressure force, $F_{S}=2 R /(1+(N-1) R)$ (see Methods), where $R$ is the reflection coefficient in intensity for an individual particle. Uniform momentum transfer is achieved when the average field intensity, $\left\langle I_{n}\right\rangle$, between particle $n-1$ and $n$, decays according to the arithmetic progression (Fig. 3a-b):

$$
\left\langle I_{n}\right\rangle=1+F_{S}\left(\frac{N}{2}+(1-n)\right), \forall n \in[2, N]
$$

where the difference in average intensity across each particle, $F_{S}$, defines the pressure force. The intensity distribution of equation (1) requires a collective phase organization described by:

$$
\Psi_{n}+\Phi_{s}=\pi-\frac{1}{2} \operatorname{asin}\left(\sqrt{\frac{1+(N-n) R}{1+(N-n)}}\right)-\frac{1}{2} \operatorname{asin}\left(R \sqrt{\frac{1+(N-n)}{1+(N-n) R}}\right)
$$

where $\Psi_{n}=2 \pi d_{n} / \lambda_{0}, \Phi_{s}$ is the phase accumulated from crossing a particle, and the residual phase, $\Psi_{n}+\Phi_{s}$, is defined modulo $\pi$ (inset of Fig. 3a). The full derivations of equations (1) and (2) are provided in the Supplementary Information. Equation (2) describes a monotonically decreasing trend (Fig. 3c), which agrees well with the experimental data when $R=0.04$ (see Methods). Remarkably, the phase distribution in equation (2) only depends on $R$ and the difference $N-n$, confirming that the residual phase between particles is locked and the $d_{n}$ are strictly bound to $\lambda_{0}$. In addition, by allowing a $\pi$-ambiguity in the total phase $\Phi_{n}+\Phi_{s}=\Psi_{n}+$ $\Phi_{s}+p_{n} \pi$ (inset of Fig. 3a), equation (2) also confirms the pseudo-crystal organization observed in Fig. 2. Importantly, equation (2) is well defined for $0<R<1$ and $n \leq N$, which stresses the existence of solutions for all experimental conditions.

The phase-locked organization described by equation (2) produces an unconventional bandgap material, whose higher-wavelength edge is enslaved to $\lambda_{0}$ and whose lower edge expands to lower wavelengths with an increasing $R$ (Fig. 3d). This bandgap feature matches remarkably well with the experimentally-observed bandgap for $R=0.04$. Bandgap enslavement at $\lambda_{0}$ can be understood by considering long systems $(N \rightarrow \infty)$ and particles close to the source, which simplifies the residual phase: $\Psi_{n}+\Phi_{s} \rightarrow \pi-\operatorname{asin}(\sqrt{R})$. We assume that the non-uniform $p_{n}$ 
can be described by a random variable with a median $\bar{p}$ and a standard deviation $\sigma_{p}$ (Fig. 3e), such that the total phase reads $\Phi_{n}+\Phi_{s} \rightarrow(\bar{p}+1) \pi-\operatorname{asin}(\sqrt{R})$. Using an effective Bloch crystal description, we obtain the location of the band edges (see Supplement):

$$
\left\{\begin{array}{l}
\lambda_{+}=\lambda_{0} \\
\lambda_{-}=\lambda_{0} \frac{(\bar{p}+1) \pi-\operatorname{asin}(\sqrt{R})}{(\bar{p}+1) \pi+\operatorname{asin}(\sqrt{R})}
\end{array}\right.
$$

where the higher-wavelength edge, $\lambda_{+}$, is enslaved to the drive wavelength independent of all other system parameters. Equation (Erreur ! Nous n'avons pas trouvé la source du renvoi.) is valid for large dispersions in $p_{n}$ (Fig. 3f). Despite increasing randomness in $p_{n}$, transmission curves with the same $\bar{p}$ emerge with similar bandgaps. The accuracy of equation (Erreur ! Nous n'avons pas trouvé la source du renvoi.) is confirmed by the full width at half maximum measured from experiment $\left(0.07 \lambda_{0}\right)$, which is close to the value obtained from equation (Erreur ! Nous n'avons pas trouvé la source du renvoi.) $\left(\lambda_{+}-\lambda_{-}=0.06 \lambda_{0}\right)$ with $R=0.04$ and $\bar{p}=1$. A qualitative understanding of band-edge enslavement can be obtained by considering the Bloch crystal description for infinite systems with uniform $\mathrm{p}_{n}$. Here, the linear decrease in intensity along the length of the structure derived in equation (1) corresponds to an infinitely long exponential decay, which must occur at the higher-wavelength band-edge transition between propagating and evanescent waves (see Supplementary Information).

\section{Phase-space attraction during dynamic self-assembly.}

We consider several benchmark cases consisting of $N=3$ particles with varied initial conditions in order to investigate transient behaviour. The transient processes are revealed by the progression in the position $(x)$ and momentum $(p)$ phase-space ${ }^{32}$, which in our system is equivalent to studying $\left[\Phi_{i}(t), v_{j}(t)\right]$ for $i$ in $\{1,2\}$ and $j$ in $\{1,3\}$. The particles' trajectories are attracted in position-space to steady states that are identical to equation (2) and in momentum-space to velocities that are uniform (Fig. 4). We find that experimental trajectories closely follow the numerical model (see Methods). 
The observation of these position and momentum-space attractors emphasizes the collapse of the system phase-space, which is a signature of dynamic self-assembly ${ }^{15}$. These attractors ultimately bestow self-healing and self-adaptive properties to the non-equilibrium system by supporting the continuous attraction towards steady state. Each of these non-equilibrium steady states are fed by their own basins of attraction that fill the position phase space, ensuring the emergence of a functionally similar bandgap structure regardless of initial placement. These basins are asymmetric, but periodic in position-space as anticipated by the spatial degeneracies observed in Fig. 2. Finally, the grey areas in Fig. 4a correspond to regions whose attractors would require particles to cross one another and are thus unreachable for the present system. Although we probe the phase-space response using 3 particles, similar dynamics occur at higher dimensions.

\section{Conclusion.}

In this work, we demonstrated a fundamentally new approach for the creation of bandgap materials that reside far from equilibrium and emerge enslaved to an external drive. Our system self-organizes into an unconventional pseudo-crystal geometry through coherentmomentum sharing. In contrast to approaches using static self-assembly ${ }^{33}$ or top-down fabrication $^{34}$ that typically result in rigid structures with fixed properties, dynamic self-assembly enables the creation of lifelike structures with dynamically imprinted properties. We observed the emergence of a phononic bandgap with the ability to self-heal and spontaneously selfadapt to changes in the source wavelength. Despite the existence of many spatial attractors, our system always self-organizes to form a transmission bandgap enslaved at its higherwavelength edge to the wavelength of the drive. Since our system relies on wave interferences, our approach could be extended to other wave systems (e.g. electromagnetic) to produce bandgap materials at different length scales.

Although dynamic self-assembly has been studied for decades, its underlying principles are only partially understood and, in particular, the role of entropy production in the emergence of order far from equilibrium is still debated ${ }^{14,35-37}$. Resolving such uncertainties is made particularly challenging due to the scarcity of physical systems that are both complex enough to 
self-organize outside of equilibrium and simple enough to be described analytically ${ }^{27}$. By solving the non-equilibrium steady-state order for an arbitrarily large number of individual elements, our work presents an ideal platform for the investigation of these unresolved theoretical issues. Finally, our demonstration of emergent phenomena through wave-based interactions illustrates a new direction for the development of non-equilibrium materials with lifelike properties that are difficult to achieve through static means. Such an approach could ultimately lead to the development of artificial self-replicating and evolving systems ${ }^{38,39}$ for the creation of synthetic living materials ${ }^{40}$ as well as collective matter displaying non-algorithmic intelligence for human-like decision making ${ }^{41}$.

Methods

\section{Acoustic setup}

The acoustic waveguide consists of a $2 \mathrm{~m}$ long, $2.2 \mathrm{~cm}$ diameter transparent acrylic tube with funnel-shaped input and output (see Supplementary Fig. ??). $20 \mathrm{~cm}$ long ramps made out of PDMS are constructed inside the waveguide to hold a $5 \mathrm{~mm}$ deep pool of viscous liquid (50\% glycerin-water solution with 0.125 wt\% Tween-20 surfactant). The ramps are designed to adiabatically shrink the waveguide mode and to make room for the liquid pool. The acoustic source consists of off-the-shelf computer speakers that are controlled through Matlab code. In all the experiments we use a $3.9 \mathrm{kHz}$ frequency to drive self-assembly, except in the reconfigurability data sets shown in Figs. $1 \mathrm{~d}$ and $2 \mathrm{~d}$ where we switch between $3.9 \mathrm{kHz}$ and 4.3 $\mathrm{kHz}$, which are two frequencies located near peaks of the speaker's emission spectrum (Supplementary Fig. 5). Transmission spectra are acquired by periodically sending a frequencychirped probe, which we register using a microphone at the end of the waveguide. Before each experiment, we obtain an empty waveguide spectrum and use it to normalize the transmission spectrum obtained during self-assembly. To improve the system performance, the output funnel of our waveguide is fitted with cotton to absorb the transmitted acoustic waves and prevent any unwanted reflections that would create stationary-wave patterns. Supplementary 
Fig. 6 shows the smooth trajectory of a single particle that is pushed along the whole length of the waveguide, illustrating that there is no stationary-wave pattern. This trajectory also demonstrates that transmission loss is minimal for our waveguide. A small amount of absorbing cotton is also introduced at the speaker input to avoid Fabry-Perot resonances due to interference between the ensemble and the speaker facet (Supplementary Fig. 7) that may appear in the transmission spectra.

\section{Meniscus particles}

Scattering particles are made from plastic straws that are cut to $10 \mathrm{~mm}$ in height and elongated into $8 \times 12 \mathrm{~mm}$ elliptic cylinders, $D_{S}=12 \mathrm{~mm}$. These cylinders are glued to a $9 \times 15 \mathrm{~mm}$ piece of rigid plastic and sealed with glue to a fitted piece of plastic on top. We glue a $5 \mathrm{~mm}$ steel rod on top of the particles, enabling magnetic loading and placement along the waveguide. $A$ photograph of a particle is shown in Supplementary Fig. 8. We estimate the particle absorption to be $\sim 1.1 \%$ (See Supplemenary Fig ??), indicating that energy dissipation is primarily attributed to viscous loss. In addition, all of the particles are characterized by their speed in an empty waveguide and a set of particles with similar speeds are chosen for the experiments. For the data sets in Figs. 1-2, the 12 particles have speeds of $[9.5,9.8,10.2,10.3$, 9.8., 9.6, 9.4, 10.8, $9.2,10.0,9.8,10.1] \mathrm{cm} / \mathrm{min}$. For the data sets in Fig. 4, the three particles chosen have speeds of $[8.7,8.6,8.8] \mathrm{cm} / \mathrm{min}$. Particles are loaded into the waveguide in the order listed and we monitor their positions using a webcam combined with a subpixel detection technique. We are required to compensate for particle inhomogeneity in Fig. 4a in order to match the locations of the attractors. We offset $D_{2}(t)$ and $D_{3}(t)$ by $5 \%$ and $2 \%$ of $\lambda_{0}$, respectively.

\section{Theory}

The system is composed of $N$ scattering particles sharing the same reflection coefficient in intensity, $R$, and distributed along the waveguide (Fig. 1a). The motion of the particles is overdamped by the large viscosity of the fluid, therefore, inertial effects are negligible and each particle satisfies: 


$$
m \frac{d^{2} x_{n}}{d t^{2}}=-\gamma v_{n}+F_{n} \approx 0
$$

where $m, x_{n}, \gamma, v_{n}$ and $F_{n}$ are the mass, position, viscous damping, velocity, and pressure force associated with the $n$th particle, respectively. The acoustic field is spatially confined to a single mode, which allows us to describe the scattering through a Transfer Matrix (TM) approach. Between particles $n-1$ and $n$ we note $A_{n}, \mathrm{I}_{n}$, and $\Phi_{n}$ as the field, intensity, and accumulated phase, respectively (see Supplementary Information). Using the TM formalism, the field and the intensity are respectively decomposed into a forward and a backward component, $\left[A_{n}^{+}, A_{n}^{-}\right]^{T}$ and $\left[I_{n}^{+}, I_{n}^{-}\right]^{T}$. At steady state the intensities at both extremities read $I_{1}=\left[1, R_{S}\right]$ and $I_{N+1}=$ $\left[T_{S}, 0\right]$ (see Fig. 1a), where $R_{S}$ is the reflection coefficient in intensity of the whole structure and

$T_{S}$ the corresponding coefficient in transmission. The pressure force exerted on the particles is responsible for a drop in average intensity across each particle, which is derived as $F_{n}=$ $\left\langle I_{n}\right\rangle-\left\langle I_{n+1}\right\rangle=\left|A_{n}^{+}\right|^{2}+\left|A_{n}^{-}\right|^{2}-\left(\left|A_{n+1}^{+}\right|^{2}+\left|A_{n+1}^{-}\right|^{2}\right)$.

\section{Numerical modelling}

The scattering properties of our particle-waveguide system are simulated in 3D with COMSOL software in order to extract the intensity reflection coefficient $R=4 \%$ with our geometry. The temporal evolution of our system is numerically investigated using a TM approach. For a given organization of the particles, we obtain the field using the TM model. The resulting forces on individual particles are then derived and the new positions are obtained from Newton's equation, neglecting inertial effects. 


\section{References}

1. Bhat, H. L. Introduction to Crystal Growth: Principles and Practice. (CRC Press, 2014).

2. Kalsin, A. M. et al. Electrostatic Self-Assembly of Binary Nanoparticle Crystals with a Diamond-Like Lattice. Science 312, 420-424 (2006).

3. Dong, A., Chen, J., Vora, P. M., Kikkawa, J. M. \& Murray, C. B. Binary nanocrystal superlattice membranes self-assembled at the liquid-air interface. Nature 466, 474-477 (2010).

4. van Blaaderen, A., Ruel, R. \& Wiltzius, P. Template-directed colloidal crystallization. Nature 385, 321-324 (1997).

5. Leunissen, M. E. et al. Ionic colloidal crystals of oppositely charged particles. Nature 437, 235-240 (2005).

6. de Nijs, B. et al. Entropy-driven formation of large icosahedral colloidal clusters by spherical confinement. Nat. Mater. 14, 56-60 (2014).

7. Grzybowski, B. A., Wilmer, C. E., Kim, J., Browne, K. P. \& Bishop, K. J. M. Self-assembly: from crystals to cells. Soft Matter 5, 1110-1128 (2009).

8. Karsenti, E. Self-organization in cell biology: a brief history. Nat. Rev. Mol. Cell Biol. 9, 255-262 (2008).

9. Shimoyama, N., Sugawara, K., Mizuguchi, T., Hayakawa, Y. \& Sano, M. Collective Motion in a System of Motile Elements. Phys. Rev. Lett. 76, 3870-3873 (1996).

10. Couzin, I. D. \& Franks, N. R. Self-organized lane formation and optimized traffic flow in army ants. Proc. R. Soc. B Biol. Sci. 270, 139-146 (2003).

11. Mateus, A. M., Gorfinkiel, N. \& Arias, A. M. Origin and function of fluctuations in cell behaviour and the emergence of patterns. Semin. Cell Dev. Biol. 20, 877-884 (2009).

12. Mora, T. et al. Local equilibrium in bird flocks. Nat. Phys. (2016). doi:10.1038/nphys3846

13. Whitesides, G. M. \& Grzybowski, B. Self-Assembly at All Scales. Science 295, 2418-2421 (2002). 
14. Kondepudi, D. \& Prigogine, I. Modern Thermodynamics: From Heat Engines to Dissipative Structures. (John Wiley \& Sons, 2014).

15. Fialkowski, M. et al. Principles and Implementations of Dissipative (Dynamic) Self-Assembly. J. Phys. Chem. B 110, 2482-2496 (2006).

16. Grzybowski, B. A., Stone, H. A. \& Whitesides, G. M. Dynamic self-assembly of magnetized, millimetre-sized objects rotating at a liquid-air interface. Nature 405, 1033-1036 (2000).

17. Grzybowski, B. A. \& Whitesides, G. M. Directed dynamic self-assembly of objects rotating on two parallel fluid interfaces. J. Chem. Phys. 116, 8571 (2002).

18. Beatus, T., Tlusty, T. \& Bar-Ziv, R. Phonons in a one-dimensional microfluidic crystal. Nat. Phys. 2, $743-748$ (2006).

19. Timonen, J. V. I., Latikka, M., Leibler, L., Ras, R. H. A. \& Ikkala, O. Switchable Static and Dynamic SelfAssembly of Magnetic Droplets on Superhydrophobic Surfaces. Science 341, 253-257 (2013).

20. Boekhoven, J. et al. Dissipative Self-Assembly of a Molecular Gelator by Using a Chemical Fuel. Angew. Chem. 122, 4935-4938 (2010).

21. Bricard, A., Caussin, J.-B., Desreumaux, N., Dauchot, O. \& Bartolo, D. Emergence of macroscopic directed motion in populations of motile colloids. Nature 503, 95-98 (2013).

22. Palacci, J., Sacanna, S., Steinberg, A. P., Pine, D. J. \& Chaikin, P. M. Living Crystals of Light-Activated Colloidal Surfers. Science 339, 936-940 (2013).

23. Martinez-Pedrero, F., Ortiz-Ambriz, A., Pagonabarraga, I. \& Tierno, P. Colloidal Microworms Propelling via a Cooperative Hydrodynamic Conveyor Belt. Phys. Rev. Lett. 115, (2015).

24. Maiti, S., Fortunati, I., Ferrante, C., Scrimin, P. \& Prins, L. J. Dissipative self-assembly of vesicular nanoreactors. Nat. Chem. 8, 725-731 (2016).

25. Vlasov, Y. A., Bo, X.-Z., Sturm, J. C. \& Norris, D. J. On-chip natural assembly of silicon photonic bandgap crystals. Nature 414, 289-293 (2001). 
26. Hynninen, A.-P., Thijssen, J. H. J., Vermolen, E. C. M., Dijkstra, M. \& Blaaderen, A. van. Self-assembly route for photonic crystals with a bandgap in the visible region. Nat. Mater. 6, 202-205 (2007).

27. Tretiakov, K. V., Bishop, K. J. M. \& Grzybowski, B. A. The dependence between forces and dissipation rates mediating dynamic self-assembly. Soft Matter 5, 1279-1284 (2009).

28. Joannopoulos, J. D., Johnson, S. G., Winn, J. N. \& Meade, R. D. Photonic Crystals: Molding the Flow of Light (Second Edition). (Princeton University Press, 2011).

29. Dholakia, K. \& Zemánek, P. Gripped by light: Optical binding. Rev. Mod. Phys. 82, 1767-1791 (2010).

30. Karásek, V. et al. Long-Range One-Dimensional Longitudinal Optical Binding. Phys. Rev. Lett. 101, (2008).

31. Frawley, M. C., Gusachenko, I., Truong, V. G., Sergides, M. \& Chormaic, S. N. Selective particle trapping and optical binding in the evanescent field of an optical nanofiber. Opt. Express 22, 1632216334 (2014).

32. Strogatz, S. H. Nonlinear Dynamics And Chaos: With Applications To Physics, Biology, Chemistry, And Engineering. (Westview Press, 2001).

33. Freymann, G. von, Kitaev, V., Lotsch, B. V. \& Ozin, G. A. Bottom-up assembly of photonic crystals. Chem. Soc. Rev. 42, 2528-2554 (2013).

34. Prather, D. W. Photonic Crystals, Theory, Applications and Fabrication. (John Wiley \& Sons, 2009).

35. Martyushev, L. M. \& Seleznev, V. D. Maximum entropy production principle in physics, chemistry and biology. Phys. Rep. 426, 1-45 (2006).

36. Ross, J., Corlan, A. D. \& Müller, S. C. Proposed Principles of Maximum Local Entropy Production. J. Phys. Chem. B 116, 7858-7865 (2012).

37. Martyushev, L. M. \& Seleznev, V. D. The restrictions of the maximum entropy production principle. Phys. Stat. Mech. Its Appl. 410, 17-21 (2014). 
38. Deplazes, A. \& Huppenbauer, M. Synthetic organisms and living machines. Syst. Synth. Biol. 3, 55 (2009).

39. Deplazes, A. Piecing together a puzzle. EMBO Rep. 10, 428-432 (2009).

40. Szostak, J. W., Bartel, D. P. \& Luisi, P. L. Synthesizing life. Nature 409, 387-390 (2001).

41. Haken, H. Principles of Brain Functioning: A Synergetic Approach to Brain Activity, Behavior and Cognition. (Springer Science \& Business Media, 2013).

Supplementary Information is available in the online version of the paper.

Author Contributions N.B. and C.R. designed and conducted experiments and performed the theoretical investigation; M.D. performed COMSOL simulations; R.Z. provided theoretical guidance; X.Z. and Y.W. guided the research. All authors contributed to writing the manuscript. 


\section{Figures}
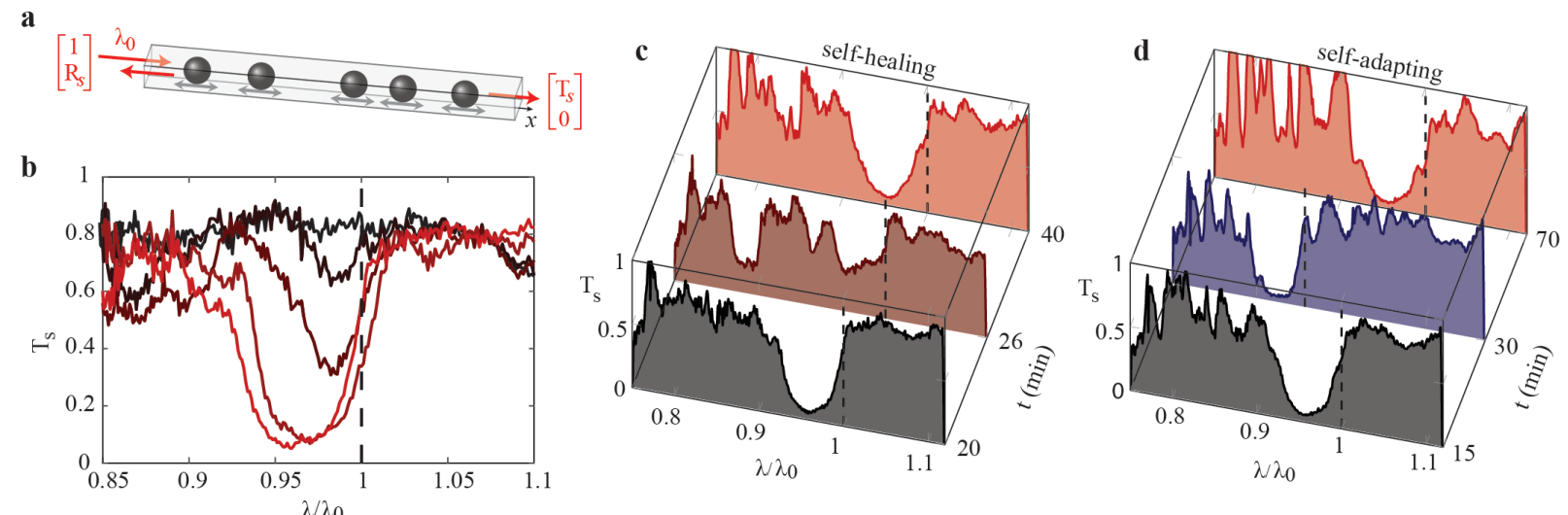

Figure 1 | Experimental observation of bandgap emergence in a non-equilibrium material. a. System diagram showing a single-mode waveguide filled with mobile scattering particles sitting in a viscous liquid. These particles are confined to move only along the $x$-direction in response to a coherent field incident from the left and with wavelength $\lambda_{0}$. The red column vectors indicate the forward and backward propagating intensities of the whole structure, where $R_{S}$ is the reflection coefficient and $T_{S}$ the transmission coefficient. b. Time evolution of a transmission spectrum showing bandgap emergence. Curves correspond to spectra taken at $t=0,5,10,20$, and $30 \mathrm{~min}$ (black to red). c. Bandgap selfhealing to a mechanical perturbation, perturbed at $t=25 \mathrm{~min}$. Spectra are taken before (black), immediately after (dark red), and following self-healing (red). d. Bandgap self-adapting to changes in the external drive wavelength. Spectra are taken when the drive wavelength is $\lambda_{0}$ (black), $0.9 \lambda_{0}$ (dark red), and returned to $\lambda_{0}$ (red). The drive is initially switched to $0.9 \lambda_{0}$ at $t=18 \mathrm{~min}$, then switched back to $\lambda_{0}$ at $t=42 \mathrm{~min}$. 


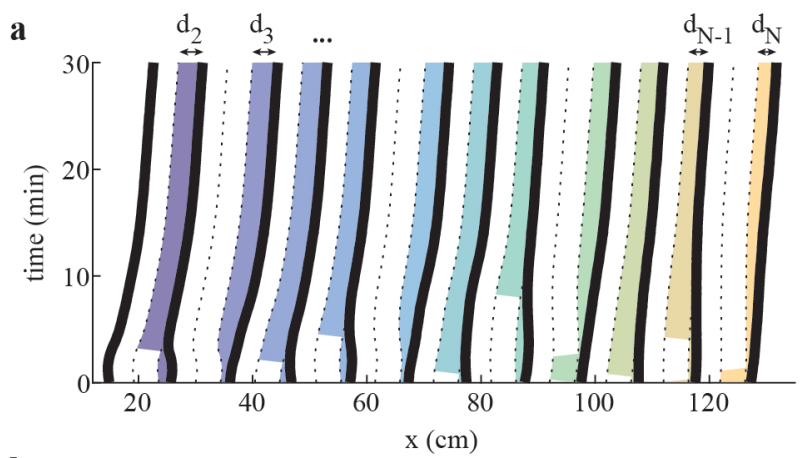

b
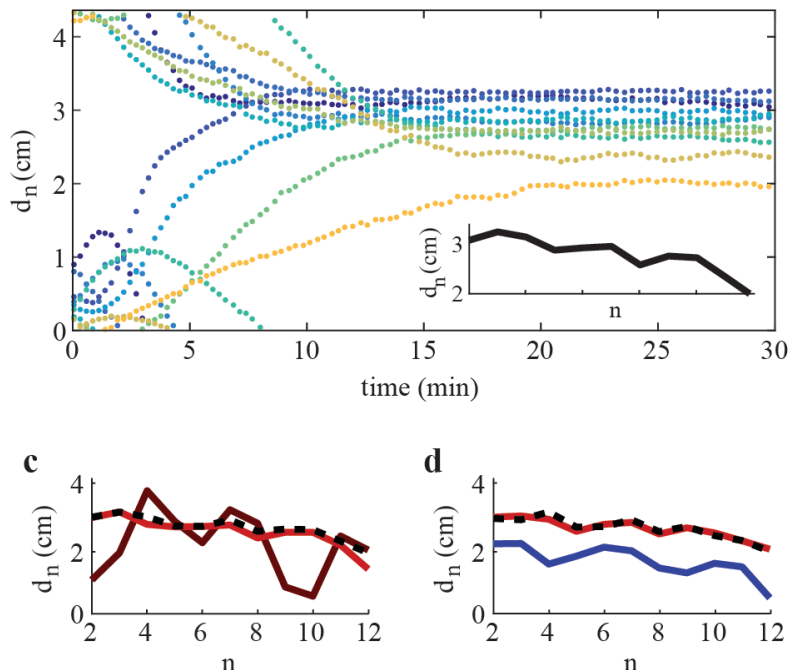

Figure 2 | Pseudo-crystal formation. a. Raw data showing the trajectory of the particle positions over time (black lines). The particles are initially regularly spaced by $12.5 \mathrm{~cm}$ along the waveguide and subsequently self-organize into a pseudo-crystal. The coloured regions label the $d_{n}$ and the dashed lines separate the different $p_{n} \lambda_{0} / 2$. b. Plot of $d_{n}$ as a function of time. Colours correspond to the regions labelled in panel a. The inset plots $d_{n}$ at steady state $(t=30 \mathrm{~min})$. c. Plot of $d_{n}$ for the self-healing data set of Fig. 1c with corresponding colours. These data show the $d_{n}$ organization before (dashed black curve, $t=20 \mathrm{~min}$ ), immediately after (dark red curve, $t=26 \mathrm{~min}$ ), and self-healed from (red curve, $t=$ $30 \mathrm{~min}$ ) perturbation. d. Plot of $d_{n}$ for the self-adaptation data set of Fig. $1 \mathrm{~d}$ with corresponding colours. These data show the $d_{n}$ organization with the source driven initially at $\lambda_{0}$ (dashed black curve, $t=15$ $\mathrm{min}$ ), after switching to $0.9 \lambda_{0}$ (dark blue curve, $t=30 \mathrm{~min}$ ), and after returning to $\lambda_{0}$ (red curve, $t=70$ min). Full time responses are provided in Supplementary Figs. 2-4. 

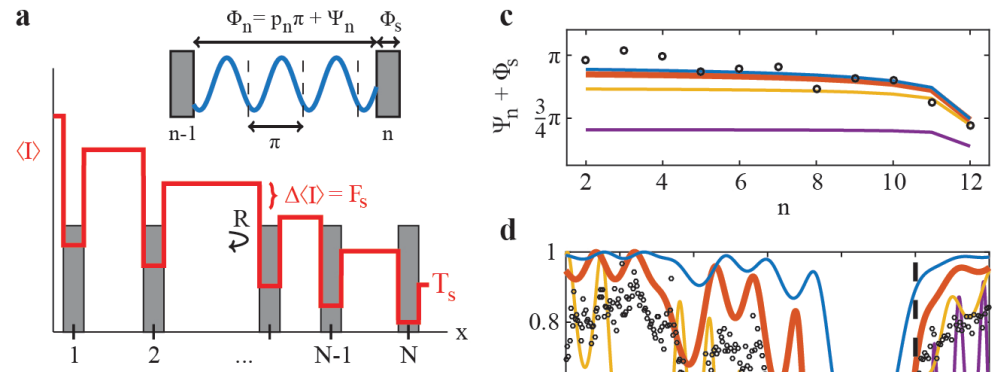

e
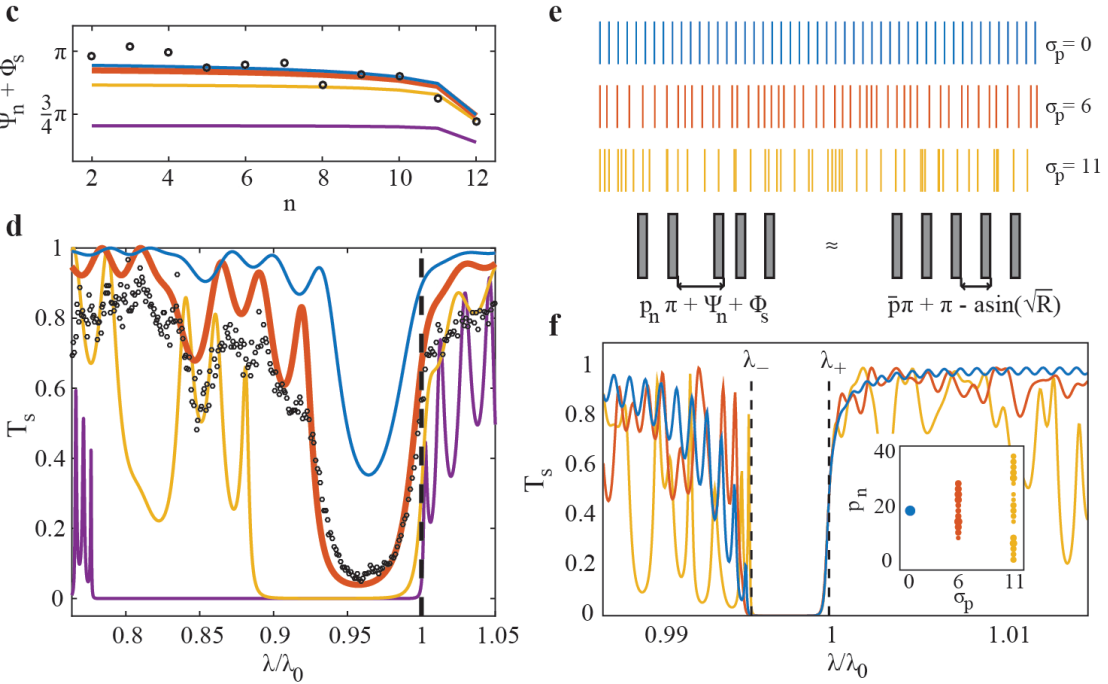

Figure 3 | Theoretical prediction of bandgap emergence out of equilibrium. a. The steady-state profile of the averaged field intensity, $\langle I\rangle$, as a function of position along the waveguide, $x$. This profile results from the self-organization of particles (grey rectangles, with individual intensity reflection coefficient $R$ ) such that wave momentum is equally shared, and the intensity drop across each particle equal to $\Delta\langle I\rangle=$ $F_{S}$. Inset labels our naming conventions used throughout the paper. b. Example steady-state intensity profile, $I(x)$, for particles separated by a non-uniform distribution of integer half wavelengths, $p_{n} \lambda_{0} / 2$. c. Phase accumulated at steady state between particles $n-1$ and $n$, modulo $\pi$, for different reflection coefficients, $R=0.01$ (blue), 0.04 (red), 0.16 (yellow), 0.64 (purple). Black circles correspond to experimental data from the inset of Fig. 2b. d. Corresponding transmission spectra from panel c, with a $p_{n}$ distribution equal to that of the experiment from Fig. 2a. Experimental data is overlaid as black circles (for $R$ estimated to be 0.04 ). e. Illustration of three $N=50$ structures with $R=0.02$ and identical $\bar{p}=20$, but increasing standard deviation, $\sigma_{p}$. These structures are modelled by equivalent Bloch crystals (see grey illustration). f. The transmission spectrum of each structure, with the same colour as in E, plotted with black dashed lines corresponding to the location of the band edges predicted analytically from equation. The inset is a histogram of the $p_{n}$ distributions as a function of $\sigma_{p}$ for the three structures shown in panel e. The size of each data point corresponds to the number of particle separations with the same $p_{n}$. 

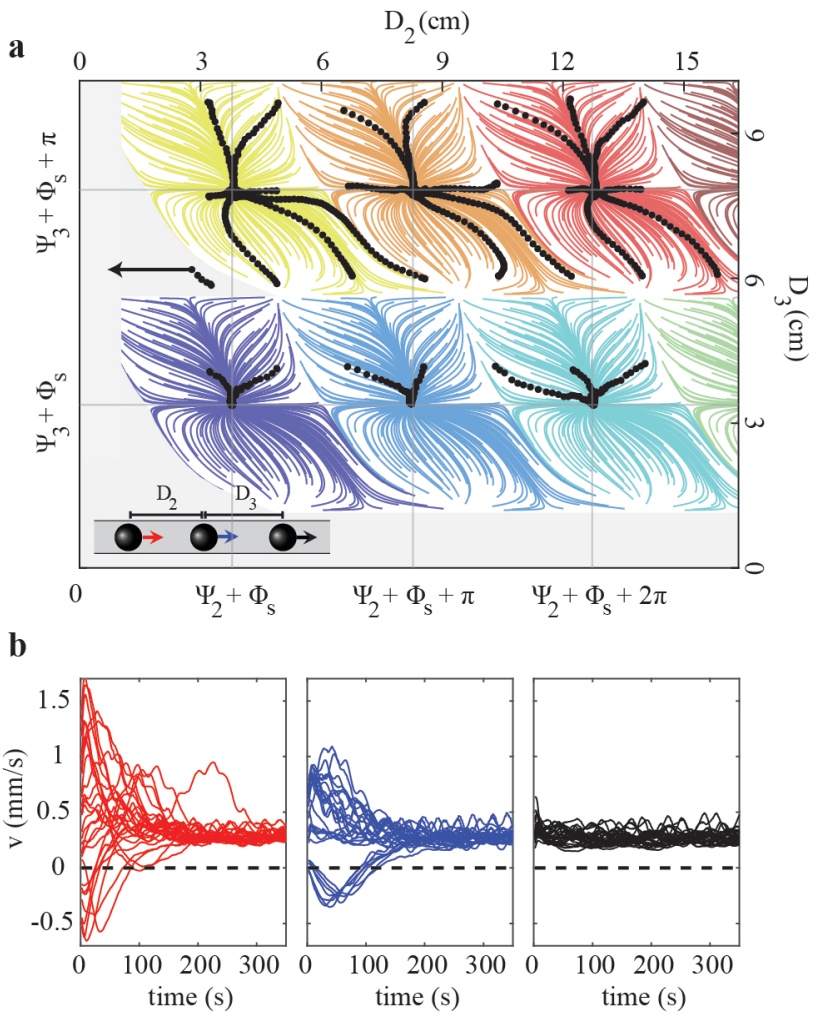

Figure 4 | Position and momentum phase-space collapse. a. Graph of the position phase-space for a $N=3$ system. The secondary axes label the corresponding real-space, with $D_{2}$ and $D_{3}$ defined in the inset. The coloured lines are the trajectories of particle separations during self-organization, obtained numerically. Different colours denote the different basins of attraction that collapse to the same steady state described by equation. The black data points are trajectories obtained experimentally from 29 different initial configurations, showing similar phase-space collapse. The greyed area represents the region where attractors force particles to collide, which is confirmed experimentally (data set with black arrow). b. The velocity of each particle (obtained using a $20 \mathrm{sec}$ running average) measured experimentally as a function of time (colours correspond to the particles as labelled in the inset of panel a). Each particle in all data sets (the colliding data set excluded) are shown converging to a common steady-state velocity. 